\title{
Diagnóstico de la situación alimentaria y nutricional de niños de tercero a quinto año básico de la comuna de la Reina, Santiago de Chile.
}

\section{Food and nutrition diagnosis of third to fifth grade school-age children from the La Reina neighborhood, Santiago de Chile}

\begin{abstract}
This article describes the nutritional status, food consumption, dietary behavior and culinary skills of third to fifth grade public school students from the La Reina neighborhood, Santiago de Chile, in order to propose healthy eating interventions. This is a cross-sectional, descriptive study in 617 children (349 males and 268 females) from third $(n=193)$, fourth $(n=202)$ and fifth grade $(n=222)$. Obesity prevalence was $30.3 \%$, greater than the $24.2 \%$ reported by National Board of Scholarships and Grants in first grade in 2015. Most of the children reported liking cooking $(66.2 \%)$, but just 20.0\% cooked mixed or seasoned vegetables, made sandwiches, or peeled or cut 4 or 5 days per week. All children (100\%) ate a late snack in the afternoon, but only $60.9 \%$ ate dinner at night. A second breakfast was eaten by $20.7 \%$ of the children and a second lunch by $35.7 \%$. Almost all children (97\%) brought money or a snack from home: $78.4 \%$ brought candies, $60.0 \%$ fruit, $50.0 \%$ dairy products and $48.4 \%$ soft drinks or juices with sugar, and $45.0 \%$ money (500 to 1000 Chilean pesos). It is necessary to educate children in healthy eating habits, with participation of parents and support from the Ministry of Education to prevent children obesity.

Key words: Nutritional status, food habits, culinary skills, nutrition education.
\end{abstract}

\section{INTRODUCCIÓN}

La obesidad infantil en Chile continúa aumentando, especialmente en niños. Los datos de la Junta Nacional de Auxilio Escolar y Becas (JUNAEB) muestran un incremento muy importante en niños de primer año básico desde un 7,5\% en 1987 hasta un 24,5\% el año 2015, último año informado por el Mapa Nutricional de JUNAEB'1 . Esto se explica por el alto sedentarismo en los niños y por sus malos hábitos alimentarios, ya descritos anteriormente ${ }^{2}$. Las últimas intervenciones realizadas en escuelas municipales muestran que la obesidad en los primeros años de educación básica está cercana al 30 por ciento, con otro 30 por ciento
Fernando Vio del R', Lydia Lera' , Carmen Gloria González', María José Fierro ${ }^{1}$, Judith Salinas ${ }^{1}$.

1. Instituto de Nutrición y Tecnología de los Alimentos, INTA, Universidad de Chile.

Dirigir correspondencia a: Fernando Vio del R. Unidad de Salud Pública, Insittuto de Nutrición y Tecnología de los Alimentos, INTA. EI Líbano 5524, Macul, Santiago. Email: fvio@inta.uchile.cl

Este trabajo fue recibido el 23 de enero de 2017. Aceptado con modificaciones el 11 de mayo de 2017. Aceptado para ser publicado el 14 de junio de 2017.

de sobrepeso, lo cual significa que 6 de cada 10 niños de educación básica están con malnutrición por exceso ${ }^{3}$. Esto es mayor a los datos de primero básico que entrega JUNAEB, en que la suma de obesidad $(24,2 \%)$ y sobrepeso $(26,8 \%)$ llega a un $51 \%$ de niños con exceso de peso'.

Existe consenso entre investigadores, educadores, personal de salud y autoridades políticas de la necesidad urgente de introducir los temas de alimentación y nutrición en las escuelas básicas del país para educar a los alumnos y sus familias en conocimientos, hábitos alimentarios y prácticas de alimentación saludable para prevenir la obesidad, contribuyendo a mejorar su salud y calidad de vida en la 
edad adulta ${ }^{4}$. Desafortunadamente no han existido avances como política pública para enseñar alimentación saludable en escuelas, especialmente dirigida al cambio de hábitos, a pesar de que la situación alimentaria y nutricional se agrava cada día más. Un avance podría ser el Plan contra la Obesidad Estudiantil de JUNAEB que se implementará el año 2017 con 50 medidas específicas para enfrentar el problema ${ }^{5}$. En este contexto y en el marco del Proyecto Fondecyt $\mathrm{N}^{\circ}$ 11407486, se realizó un diagnóstico del estado nutricional y consumo, hábitos y prácticas alimentarias en escolares de 8 a 11 años en julio de 2016 en la comuna de La Reina. El objetivo de este estudio es identificar y describir el estado nutricional, consumo, hábitos alimentarios y habilidades culinarias en alumnos de tercero a quinto año básico de una comuna de Santiago de Chile.

\section{SUJETOS Y MÉTODOS}

Diseño del estudio

Estudio descriptivo de corte transversal acerca del estado nutricional, consumo, hábitos alimentarios y habilidades culinarias en alumnos de tercero a quinto año básico de escuelas municipales de la comuna de La Reina.

\section{Sujetos}

Se consideraron las seis escuelas municipales de la comuna de La Reina, y se incluyeron todos los niños correspondientes a los cursos tercero $(n=193)$, cuarto $(n=202)$ y quinto básico $(n=222)$, los cuales sumaron 617 niños, 349 hombres y 268 mujeres. Para evaluar nivel socioeconómico se utilizó el Î́ndice de Vulnerabilidad Escolar (IVE) según pobreza en los niños asistentes a escuelas públicas?. El nivel socioeconómico variaba desde un IVE de $74,9 \%$ del Colegio Confederación Suiza y 73,6\% del Complejo Educacional La Reina, que fueron muy altos, hasta un 51,9\% en el Colegio Yangtsé, pasando por un IVE de $68,5 \%$ del Liceo Eugenio María de Hostos; $64,5 \%$ del colegio San Constantino y $63,4 \%$ de la Escuela Palestina. Con el objeto de hacer una intervención en educación en alimentación saludable posterior al diagnóstico, los colegios se dividieron en dos grupos, uno que fue intervenido (Yangtsé, Liceo Eugenio María de Hostos y San Constantino) y otro control (Confederación Suiza, Complejo Educacional La Reina y Escuela Palestina). Esta separación no se consideró para este estudio diagnóstico, que incluyó a todos los niños, tanto de los colegios intervenidos como de los controles.

\section{Estado Nutricional}

Se realizaron mediciones antropométricas de peso y talla en todos los escolares de tercero a quinto básico de los colegios intervenidos y controles, efectuado por 4 profesionales nutricionistas entrenadas y estandarizadas. Las mediciones se realizaron sin zapatos, con un mínimo de ropa en una balanza electrónica SECA ${ }^{\circledR}$, de 200 kg de capacidad y 0,1 kg de sensibilidad y la talla en centímetros con un estadiómetro marca Seca ${ }^{\circledR}$, de $220 \mathrm{~cm}$ de longitud y $0,1 \mathrm{~cm}$ de precisión. Se calculó Z-IMC $\left(\mathrm{kg} / \mathrm{m}^{2}\right)$ según IMC de la OMS 20078. Las mediciones iniciales se realizaron en junio-julio del año 2016.

\section{Encuestas a niños}

Para realizar la encuesta se utilizó un instrumento validado y publicado por el Proyecto Fondecyt No 1140748 para investigar consumo, hábitos alimentarios y habilidades culinarias en niños de 8 a 11 años con 28 preguntas: 12 de frecuencia de consumo, 10 sobre hábitos alimentarios y 6 de habilidades culinarias y gastos en la escuela ${ }^{9}$.

\section{Análisis estadístico}

Las variables fueron descritas como proporciones expresadas en \%, promedio (DE) y distribución percentilar, según tipo de variable. Se realizaron pruebas de $\mathrm{Chi}^{2}$ para la comparación de las variables por sexo, por intervenidos y controles y la prueba de Kruskal-Wallis para la comparación por colegio. El nivel de significación estadística para esta investigación se estableció como 0,05. Para el análisis se utilizó el software estadístico STATA 14.0 para Windows10.

El estudio fue aprobado por el Comité de Ética del Instituto de nutrición y Tecnología de los Alimentos (INTA) de la Universidad de Chile.

\section{RESULTADOS}

La descripción de la muestra aparece en la tabla 1, donde se observa que no hay diferencias entre los grupos control e intervenido ( $p>0.2$ ). Se muestra el estado nutricional de los alumnos de tercero a quinto año básico de la comuna de La Reina. Llama la atención el alto porcentaje de sobrepeso $(30,2 \%)$ y de obesidad (30,3\%). No se encontraron diferencias significativas entre los grupos.

En la figura 1 se muestra el estado nutricional del colegio con mayor obesidad y sobrepeso (San Constantino) con un $35,5 \%$ de obesidad y otro $32,3 \%$ de sobrepeso, comparado con el colegio con menor sobrepeso y obesidad (Yangtsé) que tiene un $27,6 \%$ de obesidad y $28,1 \%$ de sobrepeso. Es interesante destacar que el colegio San Constantino tiene un IVE de $64,5 \%$ y el Yangtsé de un 51,9\%.

En la tabla 2 se observa que al $66,2 \%$ de los niños les gusta la cocina, pero sólo un porcentaje de alrededor del 20,0\% dice saber mezclar y aliñar verduras, hacer un sándwich y pelar y picar una fruta 4 a 5 días en la semana. No se encontraron diferencias significativas entre intervenidos y controles. En la tabla 3 se aprecia el porcentaje de niños que come en cada una de las comidas del día, llegando todas a cerca del 100\%, con excepción de la cena con un $60,9 \%$, así como el lugar en el que desayunan y almuerzan. Se observa claramente que un $20,7 \%$ de los alumnos toman doble desayuno y un $35,7 \%$ comen doble almuerzo, es decir, cuando se les pregunta donde desayunan y almuerzan, responden "en la casa y colegio". Esto significa que desayunan en la casa antes de salir y luego en el primer recreo cuando lo entrega el PAE. En el caso del almuerzo, comen en el colegio y luego vuelven a almorzar al llegar a su casa. 


\section{Tabla 1}

Características de la línea base, incluido el estado nutricional, de los escolares de $3^{\circ}, 4^{\circ}$ y $5^{\circ}$ básico.

\begin{tabular}{|c|c|c|c|c|}
\hline & & $\begin{array}{c}\text { Intervenidos } \\
\mathrm{n}=334\end{array}$ & $\begin{array}{c}\text { Controles } \\
\mathrm{n}=\mathbf{2 8 3}\end{array}$ & $\begin{array}{c}\text { Total } \\
\mathbf{n}=617\end{array}$ \\
\hline & & $\%(n)$ & $\%(n)$ & (n) \\
\hline \multicolumn{5}{|l|}{ Sexo } \\
\hline & Masculino & $45,2(183)$ & 41,3 (166) & 268 \\
\hline & Femenino & $57,8(151)$ & $58,7(117)$ & 349 \\
\hline \multirow[t]{4}{*}{ Alumnos por curso } & & $\%(n)$ & $\%(n)$ & $\%(n)$ \\
\hline & Tercero & $31,4(105)$ & $31,0(88)$ & 193 \\
\hline & Cuarto & $34,4(115)$ & 30,7 (108) & 202 \\
\hline & Quinto & 34,1 (114) & $38,1(87)$ & 222 \\
\hline \multirow[t]{4}{*}{$I M C$} & & promedio $\pm \mathrm{DE}$ & promedio $\pm \mathrm{DE}$ & promedio $\pm \mathrm{DE}$ \\
\hline & Hombres & $20,2 \pm 3,8$ & $20,4 \pm 3,5$ & $20,3 \pm 3,7$ \\
\hline & Mujeres & $20,6 \pm 3,5$ & $20,5 \pm 4,1$ & $20,6 \pm 4,2$ \\
\hline & Total & $20,4 \pm 4,0$ & $20,4 \pm 3,8$ & $20,4 \pm 3,9$ \\
\hline
\end{tabular}

Estado nutricional

Hombres

Bajo Peso

Normal

Sobrepeso

Obesidad

Bajo Peso

Normal

Sobrepeso

Obesidad

Bajo Peso

Normal

Sobrepeso

Obesidad
2,2 (4)

37,7 (69)

27,9 (51)

32,2 (59)

Mujeres

3,3 (5)

36,4 (55)

$32,5(42)$

27,8 (42)

Total

2,7 (9)

37,1 (124)

29,9 (100)

30,2 (101)
1,2 (2)

34,9 (58)

30,1 (50)

33,7 (56)

3,4 (4)

40,2 (47)

30,8 (36)

25,6 (30)

2,1 (6)

37,1 (105)

30,4 (86)

30,4 (86)
1,7 (6)

36,4 (127)

28,9 (101)

33,0 (115)

3,4 (9)

38,1 (102)

31,7 (85)

28,9 (72)

Prueba $\mathrm{chi}^{2}:$ p>0,2; DE: desviación estándar

Estado Nutricional en porcentajes (\%)

Colegio San Constantino
Estado Nutricional en porcentajes (\%) Colegio Yangtsé
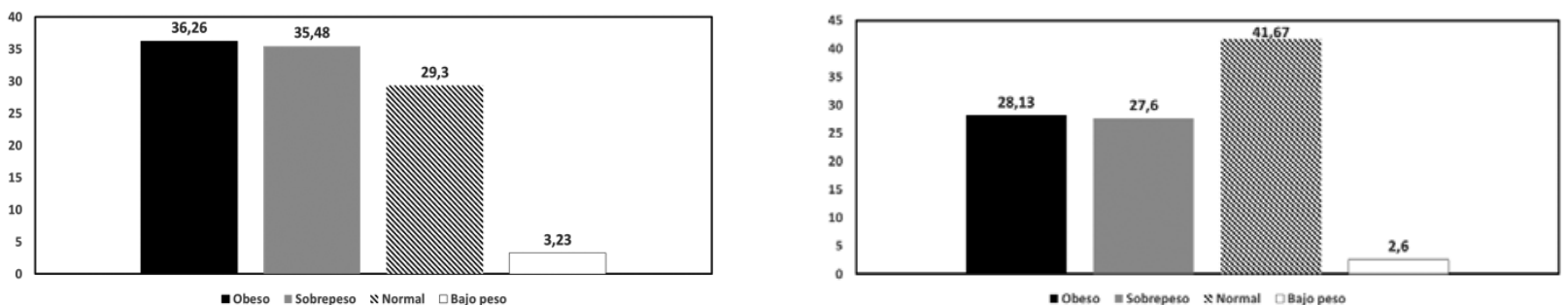

Figura 1. Comparación del Estado Nutricional Colegio San Constantino (IVE 64,5\%) y Colegio Yangtsé (IVE 51,9\%) 
Tabla 2

Habilidades culinarias de los escolares de $3^{\circ}, 4^{\circ}$ y $5^{\circ}$ básico.

Les gusta la cocina

Mezclar y aliñar verduras (días/semana)

Nunca
1
2
3
4
5

Hacer un sándwich (días/semana)

Nunca
1
2
3
4
5

Pelar y picar una fruta (días/semana)

\section{Nunca}

1

2

3

4

5

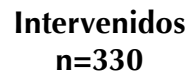

ntervenidos
$\mathbf{n}=330$
Controles

$\mathbf{n}=275$

$\%(\mathrm{n})$

$64,6(213) \quad 68,4(188)$

$30,9(102)$

$19,7(65)$

$21,5(71)$

$10,6(35)$

$3,0(10)$

$14,2(47)$

$24,2(80)$

$19,4(64)$

$20(66)$

$11,2(37)$

$3,9(13)$

$21,2(70)$

28,2 (93)

$12,7(42)$

20 (66)

10,6 (35)

$6,4(21)$

22,1 (73)
68,4 (188)

24,8 (68)

16,1 (44)

21,5 (59)

13,9 (38)

6,9 (19)

16,8 (46)

17,8 (49)

20,4 (56)

24,4 (67)

10,6 (29)

4 (11)

22,9 (63)

$25,8(71)$

$15,6(43)$

20,7 (57)

$12,0(33)$

4,4 (12)

21,5 (59)
Total

$\mathrm{n}=\mathbf{6 0 5}$

$66,28(401)$

28,15 (170)

18,05 (109)

21,52 (130)

12,09 (73)

4,80 (29)

15,40 (93)

21,32 (129)

19,83 (120)

21,98 (133)

10,91 (66)

3,97 (24)

21,98 (133)

27,11 (164)

14,05 (85)

20,33 (123)

11,24 (68)

5,45 (33)

21,82 (132)

Prueba chi²: $^{2}>0,20$

Tabla 3

Porcentaje de alumnos que come en cada una de las comidas del día y lugar dónde desayunan y almuerzan.

\begin{tabular}{|c|c|c|c|}
\hline & $\begin{array}{c}\text { Intervenidos } \\
\quad \mathbf{n}=\mathbf{3 3 0}\end{array}$ & $\begin{array}{c}\text { Controles } \\
n=275\end{array}$ & $\begin{array}{c}\text { Total } \\
\mathrm{n}=605\end{array}$ \\
\hline Comidas del día & & $\%(n)$ & \\
\hline Desayuno & $96,4(318)$ & $94,9(260)$ & $95,7(578)$ \\
\hline Colación & $96,1(317)$ & $96(263)$ & $96(580)$ \\
\hline Almuerzo & 99,7 (329) & $99,3(272)$ & $99,5(601)$ \\
\hline Once & $97,6(322)$ & $97,8(268)$ & $97,7(590)$ \\
\hline Cena & $61,5(203)$ & $60,2(165)$ & $60,9(368)$ \\
\hline Lugar en el que desayunan & & $\%(n)$ & \\
\hline Casa & 56,5 (186) & $53,9(146)$ & $55,2(332)$ \\
\hline Colegio & $18,8(62)$ & $22,9(62)$ & $20,7(124)$ \\
\hline Casa y colegio & $21,3(70)$ & $19,9(54)$ & $20,7(124)$ \\
\hline Ningún lugar & $3,3(11)$ & $3,3(9)$ & $3,3(20)$ \\
\hline Lugar en el que almuerzan & & $\%(n)$ & \\
\hline Casa & $16,4(54)$ & $19,6(54)$ & $17,9(108)$ \\
\hline Colegio & $49,9(164)$ & $41,1(113)$ & $45,9(277)$ \\
\hline Casa y colegio & 33,4 (110) & 38,6 (106) & $35,8(216)$ \\
\hline Ningún lugar & $0,3(1)$ & $0,7(2)$ & $0,5(3)$ \\
\hline
\end{tabular}


El 97,0\% de los niños lleva dinero o colación de la casa y en la tabla 4 se muestra que un $78,4 \%$ lleva golosinas dulces y un $60 \%$ frutas; un $50,0 \%$ lácteos y un $48,4 \%$ bebidas o jugos azucarados. No se encontraron diferencias significativas entre los grupos. El 45,0\% de los niños lleva dinero desde la casa y las cantidades por colegio están descritas en la tabla 5, con medianas que van de \$ 500 (0,75 US\$ dólar) a \$ 1.000 (1,5 US\$ dólar). Se encontraron diferencias significativas entre las medianas de las cantidades de dinero que llevan los niños del liceo José María de Hostos en comparación con el colegio Yangtsé (1.000 vs 500; respectivamente; $p<0,001)$ y entre la escuela Palestina y el colegio Yangtsé (1.000 vs 500; respectivamente; $p<0,001$ ).

Tabla 4

Colación que llevan los niños al colegio

\begin{tabular}{|lccc|}
\hline & $\begin{array}{c}\text { Intervenidos } \\
\mathbf{n = 3 3 0}\end{array}$ & $\begin{array}{c}\text { Controles } \\
\mathbf{n = 2 7 5}\end{array}$ & $\begin{array}{c}\text { Total } \\
\mathbf{n = 6 0 5}\end{array}$ \\
\cline { 2 - 4 } & $\%(\mathrm{n})$ & $\%(\mathrm{n})$ & $\%(\mathrm{n})$ \\
Fruta & & & $60,3(329)$ \\
Verdura & $57,8(174)$ & $63,3(155)$ & $3,1(17)$ \\
Snack salado & $3,3(10)$ & $2,9(7)$ & $17,8(97)$ \\
Lácteos & $15,6(47)$ & $20,4(50)$ & $50,6(276)$ \\
Snacks dulces & $49,5(149)$ & $51,8(127)$ & $78,4(428)$ \\
Pan & $78,4(236)$ & $78,4(192)$ & $32,8(179)$ \\
Bebidas o jugos azucarados & $28,2(85)$ & $38,4(94)$ & $48,4(264)$ \\
Bebidas o jugos sin azúcar & $48,2(145)$ & $48,6(119)$ & $21,1(115)$ \\
Agua & $22,6(68)$ & $19,2(47)$ & $4,8(26)$ \\
\hline Prueba chi': $p>0,30$. & $4.0(12)$ & $5,7(14)$ & \\
\hline
\end{tabular}

\begin{tabular}{|c|c|c|}
\hline \multicolumn{3}{|c|}{$\begin{array}{c}\text { Tabla } 5 \\
\text { Dinero que llevan de acuerdo a establecimiento } \\
\text { Dinero (pesos chilenos) }\end{array}$} \\
\hline & Promedio (DE) & $\begin{array}{l}\text { Mediana } \\
\text { (P25-P75) }\end{array}$ \\
\hline Complejo Educacional & $543,2(304,5)$ & $\begin{array}{c}500 \\
(300-1000)\end{array}$ \\
\hline Confederación Suiza & $659,8(335,9)$ & $\begin{array}{c}500 \\
(500-1000)\end{array}$ \\
\hline San Constantino & $730,4(339,0)$ & $\begin{array}{c}600 \\
(500-1000)\end{array}$ \\
\hline Liceo José M de Hostos ${ }^{\mathrm{a}}$ & $840(408,2)^{*}$ & $\begin{array}{c}1000 \\
(500-1000)\end{array}$ \\
\hline Palestino $^{\mathrm{ab}}$ & $824,7(630,9)^{*}$ & $\begin{array}{c}1000 \\
(500-1000)\end{array}$ \\
\hline Yangtséb & $590,9(308,1)^{*}$ & $\begin{array}{c}500 \\
(300-1000)\end{array}$ \\
\hline $\begin{array}{l}\text { Prueba de Kruskal-Wallis: } \\
\text { DE: Desviación Estándar }\end{array}$ & ad hoc: ${ }^{*} p<0$ & \\
\hline
\end{tabular}




\section{DISCUSIÓN}

Existe consenso en Chile y en el mundo de la necesidad de educar en alimentación saludable a los niños y sus familias desde la escuela. La estrategia de "aprender la preferencia de alimentos saludables" ha sido una de las mejor evaluadas a nivel internacional, como lo demuestran publicaciones recientes ${ }^{4}$. Para ello, es necesario tener un diagnóstico del consumo, hábitos alimentarios y habilidades culinarias que existen en la escuela, en este caso, en alumnos de tercero a quinto año básico (8 a 11 años) de una comuna de Santiago. En este trabajo se comprueba la mala alimentación de los niños en colaciones con un alto consumo de golosinas, dulces, bebidas y jugos azucarados.

En hábitos de consumo, en general comen en todos los tiempos de comida del día, excepto en la cena, donde sólo un $60,0 \%$ de los niños comen en la noche. Esto se produce por el reemplazo existente en el país de la cena por una "once" con alto consumo de pan con agregados muy poco saludables, lo cual es un hábito nacional con un $90,0 \%$ de la población chilena que toma once y sólo un $20,0 \%$ que cena $^{11-13}$. En este caso el porcentaje que cena es mayor, porque a los niños se les da de comer en la noche hasta la educación básica, para luego incorporarlos al régimen habitual de la familia, que es tomar once ${ }^{2}$. Lo grave es que un $20,7 \%$ de los niños dice tomar doble desayuno y un $35,7 \%$ doble almuerzo, lo cual ya se había encontrado en un estudio anterior donde un 22,0\% tomaba doble desayuno y un $33,3 \%$ doble almuerzo ${ }^{14}$. Esto preocupa en un país donde se está incrementando la obesidad infantil, lo cual debería ser considerado por la JUNAEB, que es la encargada del Programa de Alimentación Escolar (PAE) que entrega alimentos a los niños en las escuelas y está aplicando el Plan contra la Obesidad Estudiantil con modificaciones importantes en la dieta del PAE, junto a acciones educativas y de educación física ${ }^{5}$. Sin embargo, para enfrentar el tema del doble desayuno y doble almuerzo, es fundamental trabajar con los padres que son los que deben hacerse responsables de la alimentación de sus hijos en los hogares. Otro dato que debe ser tomado en consideración, es que un $45,0 \%$ de los niños lleva dinero con un rango que va de $\$ 500$ a $\$ 1.000$ para comprar alimentos muy poco saludables en los kioskos o fuera del colegio, lo cual también es responsabilidad de los padres, tal como se ha observado en estudios anteriores realizados por el INTA ${ }^{15,16}$.

En habilidades culinarias, un alto porcentaje de los alumnos dicen que les gusta cocinar, pero solo un $20,0 \%$ dice saber mezclar y aliñar verduras, hacer un sándwich y pelar y picar una fruta en forma frecuente, 4 a 5 días en la semana. Esto abre la posibilidad de hacer talleres prácticos de cocina en las escuelas, lo que coincide con experiencias internacionales de otros países, donde para enseñar hábitos saludables se han realizado intervenciones para enseñar a cocinar a los niños, como el "Cooking with kids program"17,18 y otras experiencias en América Latina ${ }^{19}$. Estos programas reafirman una vez más que para poder cambiar hábitos desde la niñez, incorporando a las familias, es necesario realizar actividades prácticas de "aprender haciendo", que en el campo de la alimentación saludable se expresan en talleres de cocina y utilización de huertos escolares ${ }^{20}$.

Existen interesantes experiencias internacionales que apoyan los talleres prácticos de cocina en escuelas que deberían ser replicados en nuestro país, como la del chef Jamie Oliver, quien ha desarrollado programas en el Reino Unido y otros países como Australia, cuya efectividad está siendo evaluada actualmente ${ }^{21}$.

En educación en alimentación saludable es fundamental incorporar a los padres, porque si no se hace, lo que se aprenda en la escuela se pierde en la casa. Para ello, y de acuerdo a este diagnóstico realizado en la comuna de La Reina, se iniciaron reuniones con padres de familia de los diferentes colegios considerando los siguientes temas que ellos deberían trabajar en el hogar con sus hijos: i) disminuir lo que se come en la once y reemplazarlo por alimentos saludables, o cambiar por una cena saludable con un plato de carne, pollo, pescado y ensaladas y una fruta de postre; ii) eliminar el doble desayuno o doble almuerzo; iii) como colación, enviar más frutas y lácteos y evitar golosinas, especialmente las dulces y bebidas o jugos azucarados, que deben ser reemplazada por agua; iv) además de la colación, no enviar dinero al colegio y v) aprovechando su gusto por cocinar, educar en habilidades culinarias a los niños, especialmente para preparar un plato de comida.

Sin embargo, para que el tema de alimentación saludable sea considerado prioritario en los colegios, se requiere de un fuerte apoyo del Ministerio de Educación para que incluya en sus normativas y regulaciones el tema de la alimentación saludable, ausente de las normativas escolares en la actualidad ${ }^{22}$.

En Chile, la existencia de una política de Estado en alimentación saludable para prevenir la obesidad infantil, es una necesidad imperiosa para enfrentar el tema desde diferentes ámbitos, dentro de los cuales la educación en alimentación saludable en escuelas, normada desde el Ministerio de Educación y con la participación activa de los padres, es uno de los más relevantes.

Agradecimientos: A la Corporación de Educación de La Reina, a Directores y personal docente de las escuelas municipales, que permitieron que este proyecto se llevase a efecto, en especial a Cecilia Jara, Directora de Educación y Paula Maldonado, Coordinadora Educación, Corporación Municipal de La Reina.

Financiamiento: Este estudio fue financiado por el proyecto FONDECYT No 1140748 de Educación del Concurso Regular 2014: “Elaboración, aplicación y evaluación de un programa educativo en alimentación saludable, participativo y con uso de las Tecnologías de la Información y Comunicación (TIC) para profesores, alumnos de tercero, cuarto y quinto básico y sus familias". Investigador principal: Fernando Vio del Rio. Co-investigadora: Judith Salinas Cubillos. 


\section{RESUMEN}

Se describe el estado nutricional, consumo, hábitos alimentarios y habilidades culinarias en alumnos de tercero a quinto año básico de colegios municipales de la comuna de La Reina, Santiago, Chile, para proponer intervenciones que conduzcan hacia una alimentación saludable. El estudio es descriptivo, de corte transversal en 617 niños, 349 hombres y 268 mujeres de tercero $(n=193)$, cuarto $(n=202)$ y quinto básico ( $n=222)$. El 30,3\% de los niños tenía obesidad, mayor que el $24,2 \%$ reportado por la Junta Nacional de Auxilio Escolar y Becas (JUNAEB) en primero básico el 2015. Al $66,2 \%$ de los niños les gusta cocinar, pero sólo un $20,0 \%$ mezcla y aliña verduras, hace sándwich y pela o pica fruta 4 a 5 días en la semana. Todos los niños (100\%) toman once, pero sólo un $60,9 \%$ cena en la noche. Un $20,7 \%$ toma doble desayuno y un $35,7 \%$ come doble almuerzo. Casi todos (97\%) llevaban dinero o colación de la casa. El 78,4\% llevaba golosinas dulces, $60,0 \%$ fruta, 50,0\% lácteos y $48,4 \%$ bebidas o jugos azucarados y un $45,0 \%$ lleva $\$ 500$ a $\$ 1.000$ por día al colegio. Existe la necesidad de educar en hábitos de alimentación saludable a los alumnos, incorporando padres con apoyo del Ministerio de Educación, para tener una política de Estado en prevención de la obesidad infantil.

Palabras clave: Estado nutricional, hábitos alimentarios, habilidades culinarias, educación nutricional.

\section{BIBLIOGRAFÍA}

1. National Association of School Assistance and Scholarships (JUNAEB). Chile Nutritional Map Available at: https://www. junaeb.cl/wp-content/uploads/2016/11/ Informe-MapaNutricional-2015-final.pdf

2. Vio F, Salinas J, Lera L, González CG, Huenchupán C. Food knowledge and food consumption in school-age children, parents and teachers: a comparative analysis. Rev Chil Nutr 2012; 39: 34-39.

3. Vinueza D, Lera L, Salinas J, Vio F. Evaluation of a Nutrition Intervention through a School-Based Food Garden to Improve Dietary Consumption, Habits and Practices in Children from the Third to Fifth Grade in Chile. Food Nutr Sci 2016, 7: 884-894.

4. Hawkes C, Smith TG, Jewell J, Wardle J, Hammond RA, Friel S, Thow AM, Kain J. Smart food policies for obesity prevention. www.thelancet.com. Published online February 18, 2015.

5. Obesity Students Plan. National Association of School Assistance and Scholarships (JUNAEB). https://www.junaeb. $\mathrm{cl} /$ contralaobesidad/

6. Fondecyt Project $N^{\circ} 1140748$ "Design, aplication and evaluation of an Educational program in participative healthy food with Communication, Information Technologies (ICT) for teachers, third, four and fifth grades and their families". Fondecyt Regular 2014.

7. Ministry of Education, National Association of School Assistance and Scholarships (JUNAEB). Annex N5 Targeting-Junaeb. https://www.junaeb.cl/wp-content/uploads/ 2015/06/ANNEX$\mathrm{N} \% \mathrm{C} 2 \% \mathrm{~B} 05$-Focalizacion.pdf

8. World Health Organization (WHO). The WHO 2007 SAS Macro Package. World Health Organization (WHO): Ginebra, Suiza. 2007.

9. Lera L, Fretes G, González CG, Salinas J, Vio F. Validity of an instrument for assessing food consumption, food habits and cooking skills in 8-11years old students. Nutr Hosp 2015; 31 : 1980-1991.

10. StataCorp. Stata Statistical Software: Release 14.1. College Station, TX: StataCorp LP. Statistics/Data Analysis. 2015.

11. Collect GfK. Food and vegetables consumption survey in Chile. April 2008.

12. Adimark GfK. Obesity. Chilean beliefs, attitudes and habits. July 2011.

13. Ministry of Health. National Food Consumption Survey (ENCA). Chile 2010.

14. Vio F, Lera L, González CG, Yáñez M, Fretes M, Montenegro E, Salinas J. Food consumption, dietary behavior and cooking skills in third to fifth grade school-age children and parents. Rev Chil Nutr 2015; 42: 374-382.

15. Bustos N, Kain J, Leyton B, Vio F. Changes in food consumption patterns among Chilean school children after the implementation of healthy kiosk. Arch Latinoam Nutr 2011; 61: 302-307.

16. Bustos N, Kain J, Leyton B, Olivares S, Vio F. Snacks consumed by municipal school children: choice motivations. Rev Chil Nutr 2010; 37: 178-183.

17. Lukas C, Cunningham-Sabo I. Qualitative investigation of the Cooking with Kids Program: Focus group interviews with fourth-grade students, teachers and food educators. I Educ Nutr Behav 2011; 43: 517-524.

18. Walters LM, Stacey JE. Focus on food: development of the Cooking with Kids experimental nutrition education curriculum. J Educ Nutr Behav 2009; 41: 371-373.

19. De Campo ML, Vara Messler M, Navarro A. Municipal primary school Food and Nutritional Education (EAN) program in Córdoba, Argentina. Action-participation research experience (IAP), year 2008 Diaeta (B.Aires) 2010: 28: 15-22.

20. Davis JN, Spaniol MR, Somerset S. Sustenance and sustainability: maximizing the impact of school gardens on health outcomes. Public Health Nutr 2015; 18: 2358-2367.

21. Flegs A, Herbert J, Gibbs L, Swinburn B, Keating C, Waters E, Moodie M. Methods for the evaluation of Jamie Oliver Ministry of Food program, Australia. BMC Public Health 2013; 13: 411. doi: 10.1186/1471-2548-13-411.

22. Salinas J, Correa F, Vio F Regulatory framework for promoting healthy food intake in basic schools in Chile. Rev Chil Nutr 2001; 40: 274-282. 